\title{
Size-dependent scaling of perpendicular exchange bias in magnetic nanostructures
}

\author{
Gregory Malinowski, ${ }^{1, *}$ Manfred Albrecht, ${ }^{2}$ Ildico L. Guhr, ${ }^{2}$ J. M. D. Coey, ${ }^{1}$ and Sebastiaan van Dijken ${ }^{1, \dagger}$ \\ ${ }^{1}$ CRANN and School of Physics, Trinity College, Dublin 2, Ireland \\ ${ }^{2}$ Department of Physics, University of Konstanz, D-78457 Konstanz, Germany
}

(Received 27 November 2006; published 29 January 2007)

\begin{abstract}
Size-dependent scaling is studied in exchange biased nanostructures obtained by depositing $[\mathrm{Pt} / \mathrm{Co}]_{3} / \mathrm{IrMn}$ multilayers with perpendicular magnetization onto an array of monodisperse polystyrene nanospheres with a diameter ranging from $58 \mathrm{~nm}$ to $320 \mathrm{~nm}$. We find that the exchange bias field increases with the inverse of the sphere diameter when the IrMn layer is single domain. This scaling behavior, which is favorable for further miniaturization of magnetic devices, can be understood within the context of a random field model by a statistical distribution of Mn spins at the $\mathrm{Co} / \mathrm{IrMn}$ interface.
\end{abstract}

DOI: 10.1103/PhysRevB.75.012413

Fifty years ago, Meiklejohn and Bean discovered a new form of magnetic anisotropy. ${ }^{1}$ Their discovery, called exchange bias, was a unidirectional shift of the hysteresis loop along the magnetic field axis for ferromagnetic (FM) materials in close contact with an antiferromagnet (AFM). Due to its technological applications, exchange bias coupling has generated intense experimental ${ }^{2}$ and theoretical research efforts. ${ }^{3-7}$ Nowadays, FM/AFM bilayers are incorporated in magnetic thin film devices such as magnetic spin-valve sensors, magnetic tunnel junction read heads for hard-disk drives, and magnetic random access memories. For continuous FM/AFM bilayers, variations of the exchange bias field with AFM grain size, ${ }^{5}$ AFM domain size, ${ }^{8}$ and AFM film thickness $^{9-11}$ have been measured, illustrating the theoretically predicted importance of the AFM domain size.

Although there are many reports on exchange biased nanostructures, ${ }^{12-16}$ mainly with a ferromagnetic in-plane easy axis, very few of them address in depth the effects of miniaturization on the exchange bias properties, where unique effects will set in at some critical length scales; and even the trends are controversial. While most of the nanosystems exhibit a decrease in exchange bias with size reduction, it has been recently reported for sub-100 nm NiFe-IrMn square dots, that depending on the thickness of the AFM layer, the dots could either have larger or smaller bias field than the respective continuous films. ${ }^{14}$ In addition, many studies focus on structures with dimensions larger than the AFM domain size or compare properties of single-size nanostructures with those of continuous films. Hence, a detailed understanding of size-dependent scaling of exchange bias in magnetic nanostructures that confine the AFM to a singledomain state is still lacking.

Here, we present a conclusive account of the effects of size reduction on exchange bias. In our experiments we use an original self-assembly method to systematically vary the FM/AFM structure size and AFM layer thickness. Measurements on these structures reveal a statistical enhancement of the exchange bias field in magnetic nanostructures. This enhancement, which is explained within the context of a random field model, follows a simple scaling law.

To fabricate the nanostructures, we employed dense arrays of monodisperse spherical polystyrene particles with a diameter (d) varying from $58 \mathrm{~nm}$ to $320 \mathrm{~nm}$ which were obtained by self-assembly under slow evaporation of a solvent. ${ }^{17}$ These particle monolayers were subse-
PACS number(s): 75.50.Ee, 61.46.Df, 75.70.-i, 75.75.+a

quently capped by dc magnetron sputtering of a $[\operatorname{Pt}(2 \mathrm{~nm}) / \mathrm{Co}(0.5 \mathrm{~nm})]_{3} / \operatorname{IrMn}(t$ in nm $) / \operatorname{Pt}(2 \mathrm{~nm})$ multilayer stack at room temperature, where the IrMn thickness $(t)$ was varied from $4 \mathrm{~nm}$ to $20 \mathrm{~nm}$. A decrease of the Co layer thickness towards the edges of the nanospheres results in a ferromagnetic to paramagnetic transition (at $t_{\mathrm{Co}} \approx 0.35 \mathrm{~nm}$ ) that facilitates exchange decoupling between the neighbors. ${ }^{18,19}$ Such self-assembled arrays of magnetic single domain nanocaps provide an ideal template for studying systematically the scaling behavior of coupling phenomena in magnetic nanostructures. For comparison, the films were also simultaneously deposited on plain $\mathrm{SiO}_{2}$ substrates, exhibiting perpendicular magnetic anisotropy and exchange bias. ${ }^{20,21}$ The exchange bias properties of the magnetic nanocaps and continuous films were characterized at room temperature using magneto-optical Kerr effect (MOKE) magnetometry. In the MOKE set-up focusing optics were used to reduce the diameter of the HeNe laser beam to about $40 \mu \mathrm{m}$ on the substrate. The incident angle of the laser beam was about $25^{\circ}$ so that the Kerr signal from the material deposited through the interstices is negligible.

Figure 1 shows polar MOKE hysteresis loops obtained after deposition of $[\operatorname{Pt}(2 \mathrm{~nm}) / \mathrm{Co}(0.5 \mathrm{~nm})]_{3} / \operatorname{IrMn}(10 \mathrm{~nm})$ films on particles with different diameters, and for comparison, the corresponding hysteresis loop of the continuous film, revealing a single shift towards negative applied field with an abrupt magnetization reversal (see inset of Fig. 1). The loops for the caps exhibit a double shift along the magnetic field axis, indicating a distribution of exchange-biased caps with opposite perpendicular bias directions. In fact, since the Pt/Co multilayers are deposited prior to the IrMn film, the exchange bias direction is primarily determined by the orientation of the local moment on the surface of the outermost Co layer. As we do not intentionally apply a magnetic field during deposition, the magnetization of the $\mathrm{Pt} / \mathrm{Co}$ layer will either align towards- or away from the center of the nanoparticles. The subsequent growth of IrMn on top of the Pt/Co multilayer caps biases the magnetic spin structure along these two opposite magnetization directions. The different weight in the hysteresis loops, which is particularly apparent for the particles with a diameter of $200 \mathrm{~nm}$ and $320 \mathrm{~nm}$, signifies an uneven magnetization distribution of the cap assembly. This effect is due to stray fields originating from the magnetron guns whose net out-of-plane component $(\approx 2.5 \mathrm{mT})$ favors one perpendicular magnetization state 


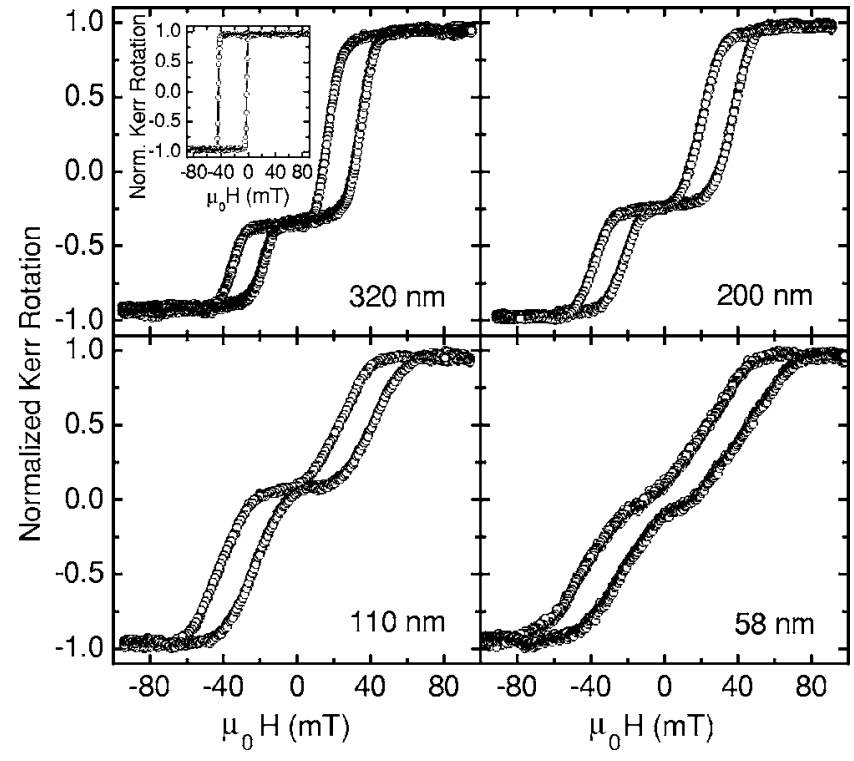

FIG. 1. Polar MOKE hysteresis loops for $[\mathrm{Pt}(2 \mathrm{~nm}) / \mathrm{Co}(0.5 \mathrm{~nm})]_{3} / \mathrm{IrMn}(10 \mathrm{~nm}) / \mathrm{Pt}(2 \mathrm{~nm})$ multilayers on arrays with different particle sizes $(320 \mathrm{~nm}, 200 \mathrm{~nm}, 110 \mathrm{~nm}, 58 \mathrm{~nm})$. The inset shows a hysteresis loop for the same multilayer grown on a flat $\mathrm{SiO}_{2}$ substrate.

over the other. ${ }^{22}$ Magnetic force microscopy (MFM) was used to image the exchange biased $320 \mathrm{~nm}$ caps at room temperature. As shown in Fig. 2 about $70 \%$ of the caps are aligned along the field direction of the magnetron guns as expected from the hysteresis loop. Interestingly, the exchange bias effect can be observed directly on individual caps. The local stray field of the magnetic tip may switch the caps with opposite magnetization orientation compared to the tip field. Usually, such a reversal event is irreversible, however, the exchange bias effect will switch the cap magnetization back, when the tip has passed the structure. These events are clearly visible in the MFM image of Fig. 2 and appear, in particular, at the outer parts of the caps where the film is thinnest, thus, exhibiting reduced bias and coercivity.

In addition to the double shift, the magnetization curves for the nanocaps are increasingly slanted for the smaller particles. This effect is due to an increase of the particle size distribution with decreasing sphere diameter yielding a larger variation of switching fields. As the coercivity of the $[\mathrm{Pt} / \mathrm{Co}]_{3} / \mathrm{IrMn}$ caps is almost independent of particle size

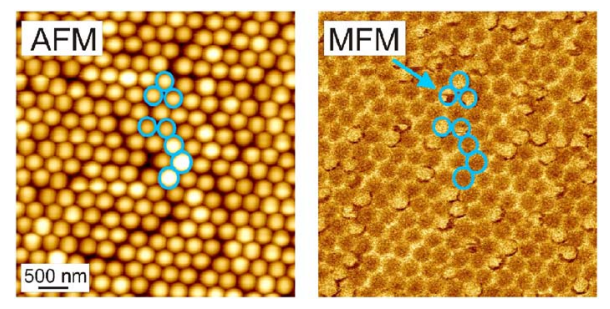

FIG. 2. (Color online) Atomic force microscope and corresponding MFM image obtained after $[\mathrm{Pt}(2 \mathrm{~nm}) / \mathrm{Co}(0.5 \mathrm{~nm})]_{3} /$ $\operatorname{IrMn}(10 \mathrm{~nm}) / \mathrm{Pt}(2 \mathrm{~nm})$ multilayer deposition onto an array of $320 \mathrm{~nm}$ polystyrene particles. The arrow indicates a tip-induced magnetization reversal event in one of the nanocaps.
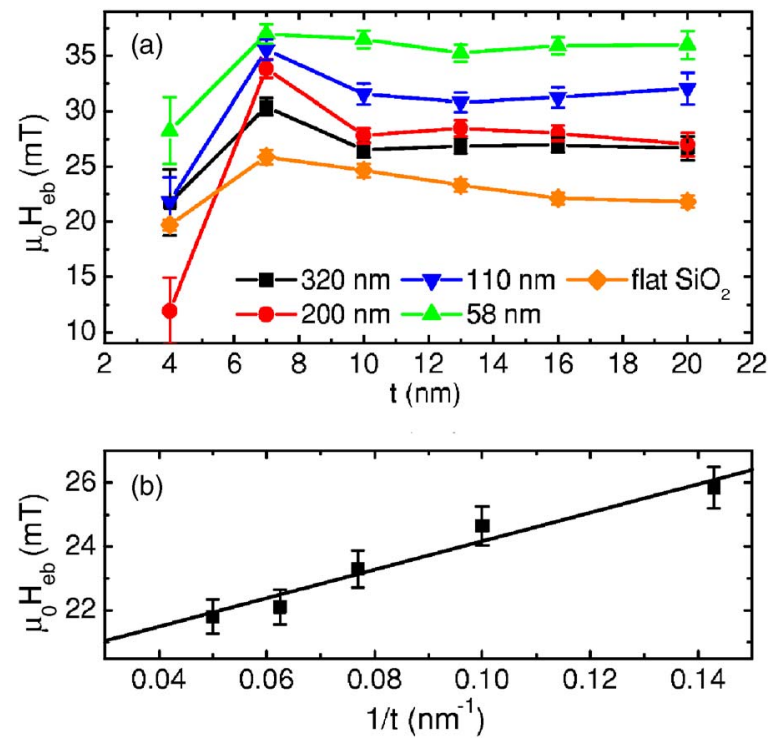

FIG. 3. (Color online) (a) Dependence of the exchange bias field on the IrMn layer thickness for $[\mathrm{Pt}(2 \mathrm{~nm}) / \mathrm{Co}(0.5 \mathrm{~nm})]_{3} /$ $\operatorname{IrMn}(t$ in $\mathrm{nm}) / \mathrm{Pt}(2 \mathrm{~nm})$ multilayers on arrays with different particle sizes and flat $\mathrm{SiO}_{2}$ substrates. (b) Exchange bias field as a function of the inverse IrMn layer thickness for films on plain $\mathrm{SiO}_{2}$ substrates. The solid line is a linear fit to the data.

(see Fig. 1), the distribution of switching fields is effected by the size dependence of the exchange bias field, which will be discussed later. Dipolar coupling between the magnetic caps plays a minor role in the switching field distribution as concluded from micromagnetic simulations, in particular, for particle sizes larger than $50 \mathrm{~nm} .{ }^{19}$

It should also be noted that the magnetization reversal process in thin films on spherical surfaces is strongly affected by the curvature of the particles. Deposition of $\mathrm{Co} / \mathrm{Pt}$ films exhibiting perpendicular magnetic anisotropy on a spherical particle results in a variation of the anisotropy axis orientation, the anisotropy strength, and the magnetic moment across the particle surface. ${ }^{19}$ Magnetic measurements on flat $[\mathrm{Pt} / \mathrm{Co}]_{3} / \mathrm{IrMn}$ multilayers, however, show that the exchange bias field increases with the angle between the applied field and the anisotropy axis. From this we infer that, contrary to unbiased particles, ${ }^{19}$ magnetization reversal is most likely initiated by domain nucleation near the center of the nanocap. While this effect might result in different bias fields for $[\mathrm{Pt} / \mathrm{Co}]_{3} / \mathrm{IrMn}$ multilayers on flat substrates and on particle arrays, it will not depend on particle size as the magnetization geometry remains the same upon scaling.

The variation of the exchange bias field $\left(\mu_{0} H_{e b}\right)$ with IrMn layer thickness $t$ is summarized in Fig. 3 for all particle sizes and for continuous films extracted from the MOKE hysteresis loops. The measurements reveal an initial increase of the exchange bias field with $t$ up to $7 \mathrm{~nm}$, which is due to an increase of the anisotropy energy of the IrMn film, leading to a larger number of pinned Mn spins. For larger IrMn film thickness, the evolution of the bias field is different for the nanostructures compared to the continuous films. Whereas the perpendicular exchange bias varies like $1 / t$ when deposited on plain $\mathrm{SiO}_{2}$ substrates [Fig. 3(b)], it re- 


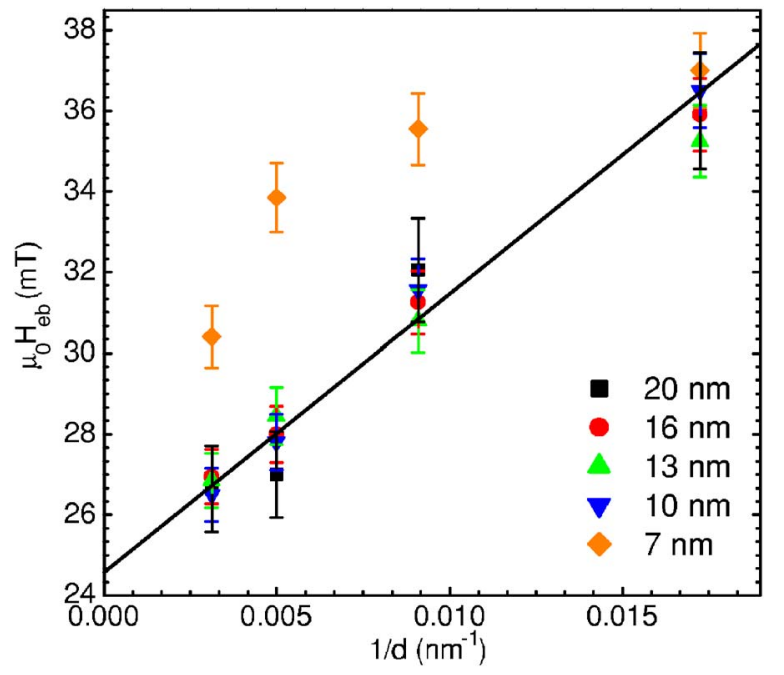

FIG. 4. (Color online) (a) Dependence of the exchange bias field on the inverse particle diameter for $[\operatorname{Pt}(2 \mathrm{~nm}) / \mathrm{Co}(0.5 \mathrm{~nm})]_{3} /$ $\operatorname{IrMn}(t$ in $\mathrm{nm}) / \operatorname{Pt}(2 \mathrm{~nm})$ caps with different IrMn layer thickness $t$. The solid line is a linear fit to the data for $t \geq 10 \mathrm{~nm}$.

mains essentially constant for the nanocaps. The $1 / t$ dependence of the exchange bias field agrees well with other experiments using $\operatorname{IrMn}$ as $\mathrm{AFM},{ }^{10,14,23,24}$ numerical simulations, ${ }^{10}$ and Malozemoff's random field model. ${ }^{4}$ In this model it is assumed that a competition between the interface coupling energy $\left(\varepsilon_{i n t}\right)$ and the domain wall energy of the $\operatorname{AFM}\left(\varepsilon_{D W}\right)$ forces the AFM into a multidomain state. For domains with dimension $D_{A F M}$ and spin-spin separation $a$, the total number of AFM spins at the interface is $N$ $=D_{A F M}^{2} / a^{2}$. A statistical distribution of these spins yields an interface coupling energy per unit area of $\varepsilon_{\text {int }}=-J_{\text {int }} / a^{2} N^{1 / 2}$ $=-J_{\text {int }} / a D_{A F M}$, with $J_{\text {int }}$ being the atomic exchange constant between the AFM and FM spins. The domain wall energy, on the other hand, can be estimated by assuming a linear spin rotation within the domain wall and an exchange constant for the AFM spins, $J_{A F M}$. For domains extending through the entire AFM film, the domain wall energy per unit area is given by $\varepsilon_{D W}=\left(\pi^{2} J_{A F M} / 2 a D_{A F M}^{2}\right) t_{A F M}$. Minimizing the total energy then gives $D_{A F M}=\left(J_{A F M} / J_{\text {int }}\right) \pi^{2} t_{A F M}$, i.e., a linear relationship between the AFM domain size and the AFM film thickness. The interface coupling energy per unit area can now be written as $\varepsilon_{\text {int }}=-J_{\text {int }}^{2} /\left(a J_{A F M} \pi^{2} t_{A F M}\right)$, which corroborates the inverse proportionality between the measured exchange bias field and the AFM layer thickness in our experiments and other exchange bias systems. ${ }^{9-11}$

The absence of the $1 / t$ variation of the exchange bias field for $[\operatorname{Pt}(2 \mathrm{~nm}) / \operatorname{Co}(5 \mathrm{~nm})]_{3} / \operatorname{IrMn}(t)$ multilayers on the nanospheres with $t \geq 10 \mathrm{~nm}$ indicates that the AFM domains are confined by the size of the particles. In this case, the AFM film is single domain and the AFM domain size scales with the diameter of the spheres instead of the IrMn film thickness. Figure 4 depicts this scaling behavior. The linear fit to the data for all particle sizes and $t \geq 10 \mathrm{~nm}$ shows that the increase of the perpendicular bias field is inversely proportional to the sphere diameter. This scaling behavior not only holds a promise for applications of the exchange bias effect in nanometer-sized devices, but also proves that the random field model accounts for the observed increase of the bias field with decreasing IrMn domain size. As stated earlier, a statistical distribution of IrMn spins at the Co/IrMn interface gives $\varepsilon_{\text {int }}=-J_{\text {int }} / a^{2} N^{1 / 2}$. Since the number of interface spins $N$ equals $D_{A F M}^{2} / a^{2}$ and $D_{A F M} \approx d$, this yields $\varepsilon_{\text {int }} \approx-J_{\text {int }} / a d$ and thus an inverse proportionality between the enhancement of the exchange bias field and particle size. From a linear fit to the data (solid line in Fig. 4) and $\mu_{0} H_{e b}=\varepsilon_{\text {int }} / m_{\mathrm{Co}} t_{\mathrm{Co}}$ it is possible to estimate the interface coupling constant. Taking $a=0.268 \mathrm{~nm}, m_{\mathrm{Co}}=1.42 \times 10^{6} \mathrm{~A} / \mathrm{m}$, and $t_{\mathrm{Co}}=1.5 \mathrm{~nm}$, we find $J_{\text {int }}=4.11 \times 10^{-22} \mathrm{~J}$, which is of the same order of magnitude as $J_{\text {int }}=7.56 \times 10^{-22} \mathrm{~J}$, the value found by Xi et al. ${ }^{23}$ In addition, extrapolation to $1 / d=0$ gives $\mu_{0} H_{e b}=24.4 \mathrm{mT}$, which is slightly larger than $19.7 \mathrm{mT}$, the exchange bias field for the continuous $[\mathrm{Pt} / \mathrm{Co}]_{3} / \mathrm{IrMn}$ multilayers on plain $\mathrm{SiO}_{2}$ substrates for $1 / t=0$. The observation of a finite exchange bias field at $1 / d=0$ indicates that the distribution of AFM interface spins is not entirely statistical. The additional net spin moment contributing to the bias field is most likely located at interface defects.

For $t=7 \mathrm{~nm}$, only the spheres with a diameter of $58 \mathrm{~nm}$ follow the $1 / d$ scaling law. In this case, the thin IrMn film is expected to break up into a multidomain state for larger sphere diameters thereby enhancing the exchange bias field. From a horizontal extrapolation in Fig. 4, it can for example be estimated that the $7 \mathrm{~nm}$ thick IrMn layer on $110 \mathrm{~nm}$ particles consists of domains with an average size of about $60 \mathrm{~nm}$. Therefore, the IrMn cap will probably consist of two domains. For larger particles the average IrMn domain size is able to increase somewhat towards its equilibrium size, which in turn leads to a reduction of the exchange bias field. However, since the IrMn film is now in a multi-domain state, the bias field for $t=7 \mathrm{~nm}$ remains larger than that of the single-domain structures. This is also clearly illustrated by Fig. 4 showing a larger exchange bias field for $t=7 \mathrm{~nm}$ than $t=10 \mathrm{~nm}$ for all particle sizes except $d=58 \mathrm{~nm}$. Finally, we note that the lateral confinement of the exchange-biased structures does not enhance thermally activated spin reversal in thicker IrMn films. This conclusion, which follows from $H_{e b}$ being independent of particle size for $t \geq 10 \mathrm{~nm}$, contrasts a report by Baltz et al. ${ }^{14}$ and is favorable for applications of thermally stable exchange-biased nanostructures.

In summary, we have demonstrated that the exchange bias field is statistically enhanced in magnetic nanostructures due to a reduction of the number of AFM spins at the FM/AFM interface. A lateral confinement of IrMn domains in $[\mathrm{Pt} / \mathrm{Co}]_{3} / \mathrm{IrMn}$ multilayers on nanoscale spheres leads to an inverse proportionality between the enhancement of the bias field and particle diameter. This scaling law provides a route for tailoring exchange anisotropy in nanostructured systems or systems with a granular AFM layer.

This work was supported by the Science Foundation Ireland as part of the CINSE program and the German Science Foundation (DFG) through the Emmy-Noether program at the University of Konstanz. G.M. acknowledges financial support from IRCSET. 
*Electronic address: malinowg@tcd.ie

${ }^{\dagger}$ Present address: VTT Micro and Nanoelectronics, P.O. Box 1000, FI-02044 VTT, Finland.

Electronic address: sebastiaan.van.dijken@vtt.fi

${ }^{1}$ W. H. Meiklejohn and C. P. Bean, Phys. Rev. 105, 904 (1957).

${ }^{2}$ J. Nogués and I. K. Schuller, J. Magn. Magn. Mater. 192, 203 (1999).

${ }^{3}$ D. Mauri, H. C. Siegman, C. S. Bagus, and E. Kay, J. Appl. Phys. 62, 3047 (1987).

${ }^{4}$ A. P. Malozemoff, Phys. Rev. B 35, 3679 (1987).

${ }^{5}$ K. Takano, R. H. Kodama, A. E. Berkowitz, W. Cao, and G. Thomas, Phys. Rev. Lett. 79, 1130 (1997).

${ }^{6}$ M. Kiwi, J. Mejía-López, R. D. Portugal, and R. Ramírez, Europhys. Lett. 48, 573 (1999).

${ }^{7}$ S. Zhang and Z. Li, Phys. Rev. B 65, 054406 (2002).

${ }^{8}$ A. Scholl, F. Nolting, J. W. Seo, H. Ohldag, J. Stöhr, S. Raoux, J.-P. Locquet, and J. Fompeyrine, Appl. Phys. Lett. 85, 4085 (2004).

${ }^{9}$ G. Malinowski, M. Hehn, S. Robert, O. Lenoble, A. Schuhl, and P. Panissod, Phys. Rev. B 68, 184404 (2003).

${ }^{10}$ M. Ali, C. H. Marrows, M. Al-Jawad, B. J. Hickey, A. Misra, U. Nowak, and K. D. Usadel, Phys. Rev. B 68, 214420 (2003).

${ }^{11}$ T. Ambrose and C. L. Chien, J. Appl. Phys. 83, 6822 (1998).

${ }^{12}$ J. Keller, P. Miltényi, B. Beschoten, G. Güntherodt, U. Nowak, and K. D. Usadel, Phys. Rev. B 66, 014431 (2002).
${ }^{13}$ J. Sort, B. Dieny, M. Fraune, C. Koenig, F. Lunnebach, B. Beschoten, G. Güntherodt, and J. Nogués, Appl. Phys. Lett. 84, 3696 (2004).

${ }^{14}$ V. Baltz, J. Sort, S. Landis, B. Rodmacq, and B. Dieny, Phys. Rev. Lett. 94, 117201 (2005).

${ }^{15}$ J. Eisenmenger, Z-P. Li, W. A. A. Macedo, and I. K. Schuller, Phys. Rev. Lett. 94, 057203 (2005).

${ }^{16}$ J. Nogués, J. Sort, V. Langlais, V. Skumryev, S. Suriñach, J. S. Muñoz, and M. D. Baró, Phys. Rep. 422, 65 (2005).

${ }^{17}$ F. Burmeister, W. Badowsky, T. Braun, S. Wieprich, J. Boneberg, and P. Leiderer, Appl. Surf. Sci. 144, 461 (1999).

${ }^{18}$ M. Albrecht, G. Hu, I. L. Guhr, T. C. Ulbrich, J. Boneberg, P. Leiderer, and G. Schatz, Nat. Mater. 4, 203 (2005).

${ }^{19}$ T. C. Ulbrich, D. Makarov, G. Hu, I. L. Guhr, D. Suess, T. Schrefl, and M. Albrecht, Phys. Rev. Lett. 96, 077202 (2006).

${ }^{20}$ S. van Dijken, J. Moritz, and J. M. D. Coey, J. Appl. Phys. 97, 063907 (2005).

${ }^{21}$ S. van Dijken, M. Besnier, J. Motitz, and J. M. D. Coey, J. Appl. Phys. 97, 10K114 (2005).

${ }^{22}$ S. van Dijken, M. Czapkiewicz, M. Zoladz, and T. Stobiecki, Phys. Status Solidi B 243, 169 (2006).

${ }^{23}$ H. Xi, J. Rantschler, S Mao, M. T. Kief, and R. M. White, J. Phys. D 36, 1464 (2003).

${ }^{24}$ J. Sort, B. Dieny, and J. Nogués, Phys. Rev. B 72, 104412 (2005). 